Pig Housing and the Environment

Occasional Publication No. 11-British Society of Animal Production 1987

edited by A. T. Smith and T. L. J. Lawrence

\title{
OBSERVATIONS ON PIG LYING AND DUNGING BEHAVIOUR IN FINISHING PENS INCORPORATING A RAISED SLATTED DUNGING AREA
}

\author{
J. D. TURNER and R. LOCKHART
}

ADAS Unit, National Agricultural Centre, Kenilworth, Warwickshire CV8 $2 L G$

\begin{abstract}
A welfare-orientated design of finishing house was built at the National Agricultural Centre Pig Demonstration Unit in 1986. This lay-out was pioneered in Sweden and allows the use of limited straw bedding on the solid-floored lying area from which pigs step up $204 \mathrm{~mm}$ to a slatted dunging area.

Internal pen length was $6.09 \mathrm{~m}$ of which $4.52 \mathrm{~m}$ was solid-floored lying area adjacent to the central access passage. The lying floor area was laid with a fall of $75 \mathrm{~mm}$ to the $1.57 \mathrm{~m}$ of dunging area next to the outer wall. The dunging area was formed of concrete slats $85 \mathrm{~mm}$ wide with $18 \mathrm{~mm}$ gaps and included a wallmounted nipple drinker. The 204-mm step up to the dunging area comprised a slat depth of $102 \mathrm{~mm}$ above a 102-mm gap. This allowed chopped straw from the lying area to pass under the overhanging slats and into the underslat scraper system. Internal pen width was $1.69 \mathrm{~m}$ from dividing wall to trough step. Trought length was $4.40 \mathrm{~m}$. Bulkhead lighting illuminated the dunging area permanently.

The room under observation was mechanically ventilated by three 'Optimavent' units discharging into 12 outlets along the two sides of the 12-pen room.
\end{abstract}

Growing pigs (gilts and boars mixed) entered the room at about $35 \mathrm{~kg}$, in groups of up to 18 per pen in six first-stage pens without trough dividers. At 45 to $50 \mathrm{~kg}$ the pigs were moved in groups of 15 to six second-stage pens with trough dividers, to finish at $85 \mathrm{~kg}$ slaughter weight. Pipeline feeding of a medium density meal with water, was available to appetite twice daily. Up to $61 \mathrm{~g}$ per pig per day of chopped barley straw was provided daily by hand.

Pigs in this 12-pen room consistently used the dunging area as a preferred lying area. At any one time, other than at feeding, 40 to $60 \%$ of pigs lay on the slats. The lying area was used for dunging and for urinating, with the part adjacent to the centre passage the most heavily fouled. Consequently, cleaning out the lying area was necessary on a daily basis. Temperature recordings have demonstrated a uniform temperature over the lying areas and dunging area. On occasions temperatures have been slightly higher in the dunging area. Air velocity checks indicated higher air speeds at pig level in the lying area of 0.2 to $0.5 \mathrm{~m} / \mathrm{s}$ compared with those in the dunging area of 0.05 to $0.3 \mathrm{~m} / \mathrm{s}$. No undue fighting or tail biting was recorded up to 27 July 1986 . It is hoped that modifications to the ventilation system will encourage a more acceptable pattern of lying and dunging, with cleaner pigs and reduced labour input. Insufficient numbers of finished pigs have been through the room to permit reliable performance data to be presented. 